\title{
Grown-in twin boundaries affecting deformation mechanisms in nc-metals
}

\author{
A. G. Frøseth, P. M. Derlet, and H. Van Swygenhoven ${ }^{\text {a) }}$ \\ Paul Scherrer Institute, Villigen $\mathrm{CH}-5232$, Switzerland
}

(Received 8 April 2004; accepted 19 October 2004)

\begin{abstract}
Molecular dynamics simulations have recently shown that the presence of grown-in twin boundaries in nc-Al promotes slip activity in the form of twin boundary migration. In this letter we investigate the effect of grown-in twin boundaries on the plastic deformation mechanism in $\mathrm{nc}-\mathrm{Ni}$ and $\mathrm{Cu}$, and show that (1) for these particular fcc metals twin boundary migration is not the favored deformation mechanism and (2) that the Schmid factors of the grown-in twin plane play a correspondingly important role. The results are explained in terms of the different ratios of the extrema of the generalized planar fault curves. (C) 2004 American Institute of Physics. [DOI: 10.1063/1.1835531]
\end{abstract}

It is known that many synthesis techniques produce nanocrystalline (nc) fcc structures $(\mathrm{Al}, \mathrm{Cu}$, and $\mathrm{Ni}$ ) with grown-in twin structures. ${ }^{1-3}$ Recent experimental observations of nc-Cu have revealed very high twin densities prior to deformation $^{2}$ - their importance on the deformation mechanism is however poorly understood. Deformation twinning was first predicted by molecular dynamics (MD) simulations of $\mathrm{Al}$ columnar structures. ${ }^{4} \mathrm{MD}$ simulations of fully 3D nc-Al systems have also shown that the presence of grown-in twins enhances plastic deformation by means of twin boundary migration, i.e., partial dislocations traveling on adjacent (111) planes. ${ }^{5}$ The onset of twin boundary migration as the major deformation mechanism was explained qualitatively in terms of the generalized planar fault (GPF) energy curves of the empirical Al potential. A similar approach has been employed to explain the nature of slip activity in defect-free nc structures for fcc materials. ${ }^{6}$ The experimental results obtained for $\mathrm{Cu}$ show however that twin boundary migration cannot be the only dominant deformation mechanism, ${ }^{3}$ where it was suggested that dislocations travel on slip planes other than the twin planes, blocked by the twins and eventually partially transmitted through them. In this letter we explain this discrepancy between $\mathrm{nc}-\mathrm{Cu}$ (experiment) and nc-Al (simulation) by showing that the effect of grown-in twins on the deformation mechanism is not the same for all fcc metals and that the resulting deformation mechanism is determined by the shapes of the GPF curves, which are substantially different for $\mathrm{Cu}, \mathrm{Ni}$, and $\mathrm{Al}$.

The $\mathrm{Cu}$ and $\mathrm{Ni}$ nc samples are generated using the Voronoi construction ${ }^{7}$ and random grain orientations, as has been extensively used in MD studies of grain boundary (GB) structure and mechanical properties. ${ }^{8-11}$ Similar to our former work on twins in Al Ref. 5 we used samples containing 15 grains with a $12 \mathrm{~nm}$ mean diameter. Different to the former studies is that we consider here within the same GB network not only samples with defect-free grains (untwinned-UT), but also samples with a given distribution of twin boundaries within the grains. In the first sample twin boundaries are introduced parallel to an arbitrary (111) plane for each grain (random twinned-RT), as was also the case for the Al samples used in Ref. 5. In the second sample the twin boundaries are introduced parallel to the (111) planes with the most optimal set of Schmid factors [high Schmid

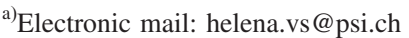

twinned-(HST)] for each grain, and finally in the third sample all twin boundaries are parallel to the (111) planes with the least optimal set of Schmid factors [low Schmidtwinned sample (LST)]. The deformation simulations were performed by applying a constant uniaxial tensile stress using the Parrinello-Rahman ${ }^{12}$ technique as explained in former papers, ${ }^{10,11}$ and similar to techniques used by other groups. ${ }^{13}$

Figure 1 displays the strain versus simulation time curves for $\mathrm{Cu}$ for an applied tensile load of $2.1 \mathrm{GPa}$ and indicates that compared to the UT-sample, the level of plasticity is approximately the same in the HST-sample while lower in the LST sample, as evidenced by the similar strain rates of the HST and UT samples $(\sim 2.5 e 8 / \mathrm{s})$ when compared to the LST sample $(\sim 6 e 7 / \mathrm{s})$. Although high compared to experiment, such strain rates are typical for simulation and an accepted caveat of the technique. ${ }^{14}$ For the UT-sample all dislocation activity is in the form of extended partial dislocations (i.e., dislocations extending from GB to GB) and predominantly on (111) planes containing high Schmid factors (exceptions to this trend have been however observed ${ }^{15}$ ). In the HST-sample dislocation activity is also predominant by means of extended dislocations, in this case however they are all parallel to the grown-in twin boundary planes but not necessarily on adjacent planes, therefore only a few events of twin boundary migration are observed. In the LST sample

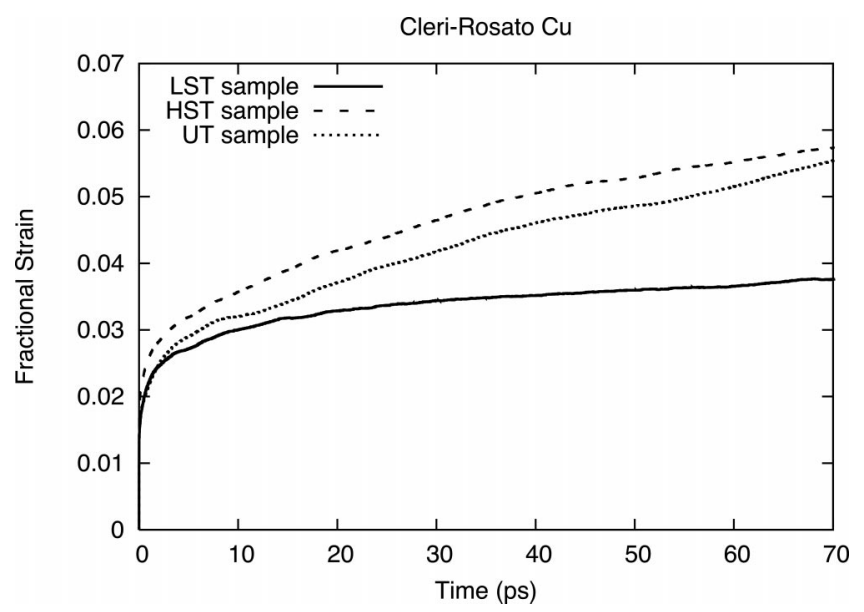

FIG. 1. Deformation curves for the nc-Cu samples: one without twins (UT), one with twins on high Schmid factor (111) planes (HST) and one with twins on low Schmid factor (111) planes (LST). 


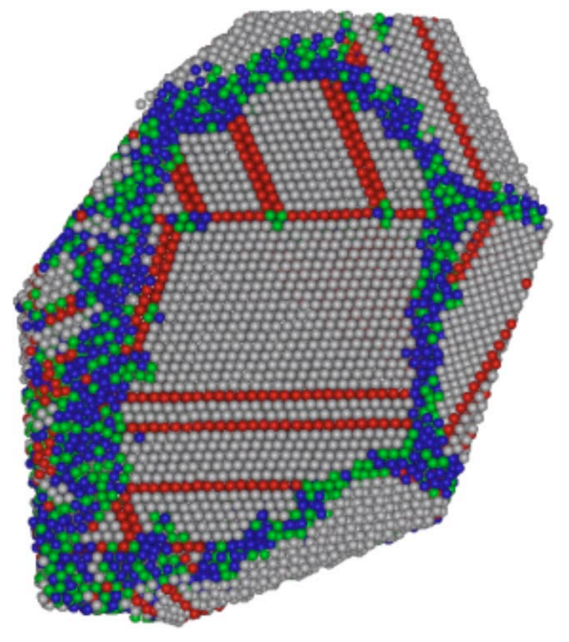

FIG. 2. Dislocations moving in regions constrained by twin boundaries in the $\mathrm{Cu}$ LST sample for an applied uniaxial tensile stress of 2.1 GPa.

partial dislocations emitted from GBs on high Schmid factor slip planes are nonparallel to the (111) twin planes and do not travel through the entire grain, indicating that the change in orientation of the slip planes across a twin boundary creates a barrier for further partial dislocation propagation. The confinement of the effective slip area explains the lower observed plasticity in the LST sample. Figure 2 shows a snapshot during deformation of the LST sample, where we have assigned colors to the atoms according to a local crystallinity classification $^{16}$ : $\quad$ grey $=\mathrm{fcc}, \quad$ red $=\mathrm{hcp}, \quad$ green $=$ other $-12-$ coordinated, and blue $=$ non-12-coordinated atoms resulting in twin (stacking) faults being represented by one (two) red hcp (111) planes.

Similarly for $\mathrm{Ni}$, in a sample with a random distribution of twin planes and deformed with an uniaxial stress of 2.6 $\mathrm{GPa}$ (the same value that was used for former UT nc Ni samples ${ }^{11}$ ), both extended dislocations and twin boundary migration were observed: single partial dislocations move both on slip planes parallel and at angles to twin boundaries, generally on planes with high Schmid factors. The grown-in twin boundaries are also observed to act as barriers for the partials that are traveling on slip planes nonparallel to the grown-in twin planes.

For a RT-Al sample however, it was shown that in grains where the grown-in twins are oriented along a (111) plane with an optimal set of Schmid factors, twin boundary migration was observed as the dominant deformation mechanism and not partial or full dislocations traveling on other slip planes. ${ }^{5}$ This observation for $\mathrm{Al}$ could be understood in terms of the GPF curves. The GPF curves represent the energy cost of a $[11 \overline{2}]$ rigid slip of two semi-infinite blocks of closepacked planes with respect to each other, giving both an unstable and stable planar fault surface energy density, the former of which gives an energy barrier to the nucleation of the planar fault. For more details of such rigid slip associated with partial and full dislocations see Ref. 6. When the initial configuration contains a single twin boundary, a rigid slip on a (111) plane adjacent to the twin boundary will result in the twin boundary migrating one (111) plane with respect to the initial configuration. The corresponding energies for the stable and unstable twin migration configurations are $\gamma_{u t m}$ and $\gamma_{t m}$. Figure 3 displays the GPF curves for $\mathrm{Cu}$, and $\mathrm{Ni}$, using the second moment tight-binding potentials of Cleri
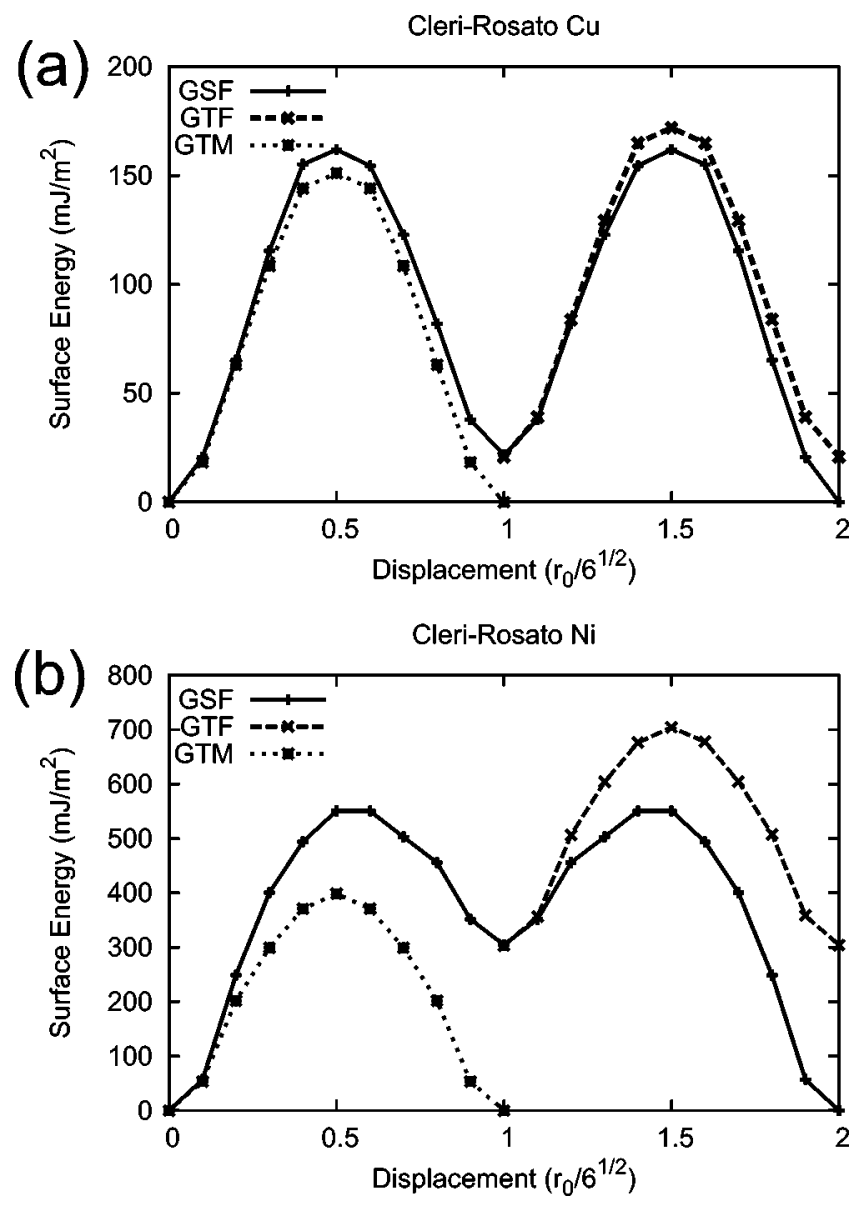

FIG. 3. Generalized planar fault (GPF) energy curves for (a) $\mathrm{Cu}$ and (b) Ni.

and Rosato ${ }^{17}$ for $\mathrm{Cu}$ and Ni. For comparison to the Al GPF curves see Ref. 5.

Looking at these GPF curves, it is now possible to explain the difference in dislocation activity in the $\mathrm{Al}, \mathrm{Ni}$, and $\mathrm{Cu}$ samples in the presence of twin boundary planes prior to deformation. For Al, $\gamma_{u s f} / \gamma_{u t m} \sim 3,{ }^{5}$ and twin boundary migration becomes preferable over single partial dislocation nucleation. Contrary to this, the ratio $\gamma_{u s f} / \gamma_{u t m}$ for $\mathrm{Cu}$ [Fig. 3(a)] is $\sim 1$ and for Ni [Fig. 3(b)] is $\sim 1.3$. Therefore both twin boundary migration and extended partial dislocations will be observed simultaneously on high Schmid factor slip planes. For instance in HST Cu where all grown-in twins are along (111) planes with the optimal set of Schmid factors, all dislocations will be emitted on planes parallel to the grown-in twins, but not necessarily adjacent to twin planes. On which plane the partial will be emitted from the grain boundary will depend on the stress distribution in the GBs, as has been shown in former studies of dislocation emission from GBs. ${ }^{11}$ On the other hand, when all grown-in twins are along (111) planes with the least optimal set of Schmid factors as is the case for the LST sample, little twin boundary migration will be observed with the twin planes acting as barriers for dislocation propagation.

The current observations have to be put into the context of the possible high-stress/short-time artefacts of atomistic simulations. ${ }^{6,18,19}$ In $\mathrm{Cu}$ and $\mathrm{Ni}$ simulations of defect free grains, only extended partial dislocations emitted from the GBs have been observed up to a mean grain size of $50 \mathrm{~nm}$ for $\mathrm{Cu}$ (Ref. 9) and $30 \mathrm{~nm}$ for $\mathrm{Ni}^{20}{ }^{20}$ That no full dislocations are observed in tensile simulations have been explained by 


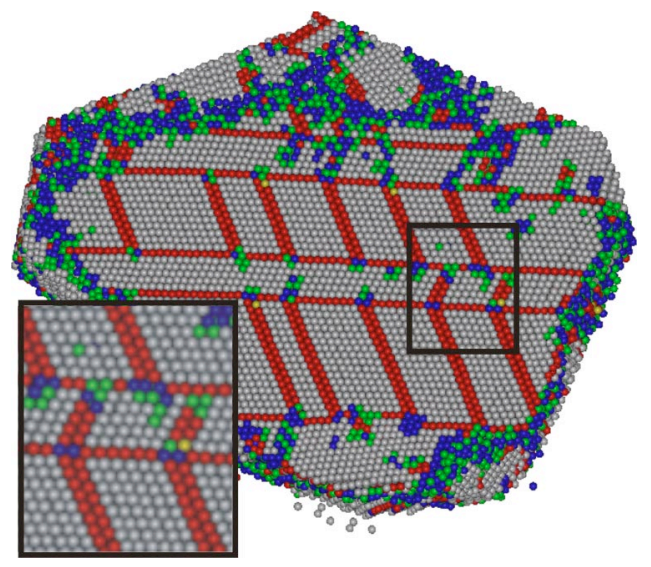

FIG. 4. Dislocations being transmitted across twin boundaries at high stress in RT Cu sample for an applied uniaxial tensile stress of 4.0 GPa.

the high value of the ratio between the unstable and stable stacking fault energies ${ }^{6}$ and the short time of the simulation. It was shown that by applying a higher stress in the simulation the energy barrier for full dislocation activity within the simulation time could be overcome, and full dislocations and twinning can be observed even in very small grains such as $12 \mathrm{~nm}$ for Ni. Motivated by these former results we increased the stress by a factor of 2 for the LST Cu sample. Figure 4 shows a section of this sample demonstrating that some of the partial dislocations are, in part, transmitted across the twin boundaries, either onto the mirror image (111) plane or onto a (100) plane via a [100] dislocation. As demonstrated in the inset of Fig. 4, a residual Burgers vector remains in the twin plane, as evidenced by the presence of the green-blue atoms at the twin plane after transmission, creating a stress concentration in the twin plane. However, in terms of the abovementioned possible high-stress/short-time artefacts, it is not excluded that in a real sample within experimental deformation times, full dislocations are emitted from the grain boundaries instead of partial dislocations and are also hindered by the twin boundaries being eventually transmitted after dissociation, as has been recently observed experimentally in a twin dominated $\mathrm{Cu}$ microstructure. ${ }^{2}$

In summary, we have shown that the presence of grown-in twins in nc-fcc metals can change the deformation mechanism, but that the effect is not the same for all fcc metals and can be explained by the relative values of the extrema of the generalized planar fault energy curves. For the case of $\mathrm{Cu}$ and $\mathrm{Ni}$, which have twin migration energy barriers that are not so different from their partial dislocation energy barriers [unlike the case for $\mathrm{Al}$ (Ref. 5), twin boundary migration will not become dominant. The current simulations also demonstrate the importance of the Schmid factor in the activation of slip systems, even at such small grain sizes. The present work demonstrates once more the usefulness of the GPF curves in understanding atomistic simulations of the mechanical properties of fcc nc systems.

The authors would like to acknowledge the support of the Swiss-FN (Grant No. 200021-100055/1).

${ }^{1}$ J. R. Weertman, Mechanical Behavior of Nanocrystalline Metals in Nanostructured Materials: Processing, Properties and Potential Applications (Willian Andrew, Norwich, NY, 2002).

${ }^{2}$ M. Chen, E. Ma, K. J. Hemker, H. Sheng, Y. Wang, and X. Cheng, Science 300, 1275 (2003).

${ }^{3}$ L. Lu, Y. Shen, X. Chen, L. Qian, and K. Lu, Science 304, 422 (2004).

${ }^{4}$ V. Yamakov, D. Wolf, S. R. Phillpot, A. K. Mukherjee, and H. Gleiter, Nat. Mater. 1, 45 (2002).

${ }^{5}$ A. Frøseth, H. Van Swygenhoven, and P. M. Derlet, Acta Mater. 52, 2259 (2004).

${ }^{6}$ H. Van Swygenhoven, P. M. Derlet, and A. G. Frøseth, Nat. Mater. 3, 399 (2004).

${ }^{7}$ G. Z. Voronoi, J. Reine Angew. Math. 134, 199 (1908).

${ }^{8}$ H. Van Swygenhoven, D. Farkas, and A. Caro, Phys. Rev. B 62, 831 (2000).

${ }^{9}$ J. Schiøtz and K. W. Jacobsen, Science 301, 1357 (2003).

${ }^{10}$ H. Van Swygenhoven, P. M. Derlet, and A. Hasnaoui, Phys. Rev. B 66, 024101 (2002).

${ }^{11}$ P. M. Derlet, H. Van Swygenhoven, and A. Hasnaoui, Philos. Mag. 83, 3569 (2003).

${ }^{12}$ M. Parrinello and A. Rahman, J. Appl. Phys. 52, 12 (1981).

${ }^{13}$ V. Yamakov, D. Wolf, S. R. Phillpot, A. K. Mukherjee, and H. Gleiter, Nat. Mater. 3, 43 (2004).

${ }^{14}$ P. M. Derlet, A. Hasnaoui, and H. Van Swygenhoven, Scr. Mater. 49, 629 (2003).

${ }^{15}$ H. Van Swygenhoven, M. Spaczer, and A. Caro, Acta Mater. 47, 3117 (1999).

${ }^{16}$ J. D. Honeycutt and H. C. Andersen, J. Phys. Chem. 91, 4950 (1987).

${ }^{17}$ F. Cleri and V. Rosato, Phys. Rev. B 48, 22 (1993).

${ }^{18}$ H. Van Swygenhoven, P. M. Derlet, and Z. Budrovic, Z. Metallkd. 10, 1106 (2003).

${ }^{19}$ P. M. Derlet, A. Hasnaoui, and H. Van Swygenhoven, Scr. Mater. 49, 629 (2003).

${ }^{20}$ M. J. Caturla, H. Van Swygenhoven, and P. M. Derlet (unpublished). 